\title{
La financiación pública de la innovación empresarial en Boyacá Colombia: un estudio de caso
}

\author{
Sandra Lorena Díaz Goyeneche* \\ José Luis Cruz Vásquez** \\ Claudia Jessenia Becerra Gualdrón ${ }^{* * *}$
}

Fecha de recepción: 2 de septiembre de 2020

Fecha de aprobación: 19 de enero de 2021

Resumen: El departamento de Boyacá actualmente presenta bajos niveles de innovación, lo cual repercute directamente en su crecimiento económico y en la sostenibilidad de sus empresas. Este artículo estudia el caso de dos empresas que tuvieron acceso directo a recursos públicos para la promoción de la innovación, con el objetivo de analizar la influencia de dicha inversión sobre el desempeño general e innovativo de la empresa y su aporte al crecimiento económico del departamento. Se desarrollaron tres etapas empleando la metodología de estudio de caso: en primer lugar se elaboró un modelo de análisis que permitiera la construcción de indicadores de tipo cuantitativo y cualitativo; en segundo lugar se aplicó este modelo en dos empresas que tuvieron acceso a recursos a través de convocatorias públicas para la ejecución de proyectos de innovación. Y en último lugar se presentaron algunas recomendaciones a los actores involucrados para aumentar la eficiencia de la inversión de estos recursos.

Palabras clave: innovación; investigación y desarrollo; recursos públicos; crecimiento económico; comportamiento económico.

Clasificación JEL: L10, O32, O38.

Como citar este artículo:

Díaz-Goyeneche, S., Cruz-Vásquez, J., \& Becerra Gualdrón, C. (2021). La financiación pública de la innovación empresarial en Boyacá Colombia: un estudio de caso. Apuntes del Cenes, 40(71). Págs. 253 - 273. https://doi. org/10.19053/01203053.v40.n71.2021.11674

\footnotetext{
Administrador de empresas, especialista en Alta Gerencia en Mercadotecnia de la Universidad Pedagógica y Tecnológica de Colombia UPTC. Iorenadiazg2705@gmail.com (iD https://orcid.org/0000-0002-9702-0088

** Economista, magíster en Economía, docente de la Escuela de Economía de la Universidad Pedagógica y Tecnológica de Colombia UPTC. jlcruzv@gmail.com (iD https://orcid.org/0000-0002-5432-6909

*** Economista, magíster en Economía, candidata a PhD Wageningen University and Research. clajebegu@gmail.com (i) https://orcid.org/0000-0001-5148-906X
} 


\title{
Public Financing of Business Innovation in Boyacá Colombia: A Case Study
}

\begin{abstract}
Boyacá state currently presents low levels of innovation affecting directly on its economic growth and sustainability of its companies. This article studies the case of two companies that had direct access to public resources for the promotion of innovation, with the aim of analyzing the influence of said investment on the general and innovative performance of the company and its contribution to the economic growth of the state. Through the case study methodology, three stages were proposed, firstly, an analysis model was built that allowed the construction of quantitative and qualitative indicators; secondly, this model was applied in two companies that had access to resources through public calls for the execution of innovation projects. Third and last, some recommendations were presented to the stakeholders to increase the efficiency of the investment of these resources.
\end{abstract}

Keywords: innovation; research and development; public resources; economic growth; economic behaviour.

Clasificación JEL: L10, O32, O38 


\section{INTRODUCCIÓN}

En los últimos años la innovación ha adquirido significativa importancia en la construcción de las políticas públicas nacionales y regionales, desde el momento en que el concepto se relacionó directamente con economías líderes en el mundo y se convirtió en hoja de ruta para buscar el crecimiento económico de una región.

La innovación es definida como un proceso colectivo, enormemente incierto y acumulativo, dirigido a la generación y aplicación de nuevos conocimientos. Su importancia radica en la condición de variable indispensable para tener una ventaja competitiva, requiere además que se fomenten las buenas relaciones entre las áreas, la cultura de la competitividad, el espíritu innovador, el desarrollo de creatividad y la generación de liderazgo para la realización efectiva de proyectos que se transforman en oportunidades de mayor y mejor crecimiento, en bienestar social y en una ampliación de los horizontes.
La escuela neoclásica maneja la teoría según la cual los factores tradicionales de producción -capital, tierra y trabajo- son los causantes del crecimiento económico $\mathrm{y}$, por lo tanto, de la dinámica del sistema capitalista en su conjunto. Esta teoría es refutada por Schumpeter (1939), pues argumenta que la producción capitalista es principalmente influenciada por los procesos de innovación tecnológica y de transformación constante. El Estado, mediante políticas públicas, desempeña un papel fundamental para fomentarla en el ámbito empresarial con un crecimiento económico inclusivo. En los países que han alcanzado crecimiento liderado por la innovación, el Estado asumió el liderazgo en la realización de inversiones orientadas al financiamiento para las empresas en etapas tempranas a través de acciones de capital de riesgo.

La relación entre innovación y crecimiento es innegable. Nuevos productos, nuevos procesos y nuevas formas de organizar la producción, cambian cuantitativa y cualitativamente la estructura de la economía y de la sociedad. 
Es un elemento central en la estrategia de desarrollo, definido como un proceso dinámico de interacción que une agentes que trabajan guiados por incentivos de mercado (como las empresas) y otras instituciones (como los centros públicos de investigación y las instituciones académicas) que actúan de acuerdo a estrategias y reglas que responden a otros mecanismos y esquemas de incentivos. (CEPAL, 2006)

A pesar de la importancia de la inversión pública en innovación para el crecimiento económico de la región, hasta ahora no existe un sistema de información oficial, centralizado y amigable, sobre la evolución y composición del gasto en innovación. Estos sistemas de información resultan clave para informar etapas prioritarias, tales como la planificación y priorización sectorial, selección de proyectos y asignación presupuestaria, monitoreo y evaluación. Por falta de datos estandarizados sobre la composición de dicho gasto, Boyacá enfrenta grandes desafíos para cerrar las brechas de productividad y mantener las tasas de crecimiento registradas en la última década. Uno de los aspectos clave para impulsar la productividad tiene que ver con el grado en que las empresas logran incorporar innovación tecnológica a sus procesos productivos. ¿Qué tan eficiente es la priorización de funciones del gasto en inversión pública con respecto a las brechas de infraestructura sectoriales? ii) ¿Qué tan eficiente es la distribución del gasto? En este contexto, el objetivo de este documento es analizar si los instrumentos públicos (subvenciones y deducciones fiscales) de los últimos cinco años contribuyeron a impulsar el crecimiento económico en Boyacá, tomando como base el análisis de caso de dos empresas beneficiadas con recursos de cofinanciamiento público, a fin de aportar a la generación de conocimiento y establecer una línea base de información que sirva como punta de lanza a investigaciones más profundas con miras al desarrollo integral de estrategias propuestas por los actores económicos de la región.

El documento está dividido en cuatro secciones. En la primera se presenta una revisión de la literatura en torno a la política pública, los instrumentos de fomento a la innovación y el crecimiento económico. En la segunda parte se describen la metodología y las variables cualitativas analizadas, se exponen los principales resultados del análisis y se responden los interrogantes objeto de estudio: cuánto se invierte en Boyacá, qué nivel del Gobierno invierte y en qué se invierte. En la tercera parte se realiza el análisis derivado de la información, y en la cuarta parte se exponen algunas conclusiones derivadas del trabajo de investigación.

\section{FOMENTO A LA INNOVACIÓN Y CRECIMIENTO ECONÓMICO}

La inversion pública se define como la "formación bruta de capital fijo" o "adquisición neta de activos no financieros" del Gobierno durante un período contable específico. La innovación es un 
argumento relevante en los debates económicos por su efecto potencializado en el crecimiento y desarrollo de una región. Schumpeter (1939) la considera como el impulsor en la generación de nuevos o mejorados bienes y procesos, lo cual a su vez se presenta como un carácter inherente a la empresa al ser desarrollada por un empresario innovador para adquirir mayores beneficios. De igual forma, Romer (1990) señala dos efectos del desarrollo de conocimientos: en primer lugar posibilita la producción de un nuevo o mejorado bien; en segunda instancia contribuye a aumentar el stock de conocimientos disponibles; las empresas que realizan innovación alcanzan un margen de beneficios superior al que perciben las empresas que no lo hacen. El margen de beneficios atrae a nuevas empresas del sector, lo que conduce a aumentos en el tamaño de la industria hasta el punto en el que elimine el diferencial del margen de utilidades obtenidos por las empresas. Por consiguiente, es importante que las empresas mantengan una constante inversión en investigación y desarrollo (I+D) para así garantizar su permanencia con productos cada vez más especializados en las necesidades y preferencias del consumidor final.

Sin embargo, algunas situaciones del mercado no permiten que las empresas se apropien de la totalidad del beneficio de una innovación. En gran parte porque el conocimiento posee características de un bien público. Además, por definición, la información cumple la condición de ser indivisible, es decir, cuando la empresa coloca un nuevo producto en el mercado, este pude ser reproducido sin sobrecostos y de esta forma el empresario innovador pierde el poder de monopolio sobre el producto o servicio (Romer, 1990). Estas fallas de mercado llevan a que la intervención del Estado sea necesaria, para que, en condiciones de competencia e incertidumbre, el nivel de inversión en (I+D) no sea inferior al requerido para alcanzar el óptimo social (Arrow, 1962). Adicionalmente, y como lo sugiere la Organización Mundial de la Propiedad Intelectual (OMPI) en su más reciente publicación del Índice Mundial de Innovación 2020, la PI se debe ver como un activo para financiar e impulsar la innovación, ya que posee una relevancia directa en la forma en que las empresas pueden desbloquear las inversiones que realizan a través de los mercados financieros. En este sentido, el foco de la intervención del Estado sería facilitar la maximización del retorno de la PI, a fin de lograr un mejor conocimiento, flujo de información y acceso a mecanismos financieros, lo que incentiva la creación de nuevas ideas e impulsa la actividad innovadora, que mejora el crecimiento económico (Cornell University, INSEAD \& WIPO, 2020).

En este sentido, la innovación en sí misma no es una garantía para el desarrollo regional. Requiere tomar riesgos elevados, puesto que no es posible tener una idea anticipada de los resultados, lo que hace que se desaceleren los proce- 
sos de innovación, debido a los temores de los diferentes actores a asumir este riesgo. Por ende, para fomentar y crear un contexto propicio para innovar, son necesarias políticas públicas adecuadas en materia de regulación financiera, tributaria, contractual y de propiedad intelectual. La OCDE (2014) reconoce la innovación dentro del sistema empresarial como un elemento que fomenta el desarrollo de nuevas actividades económicas, eleva los niveles de productividad y contribuye a la generación de empleos. $\mathrm{Si}$ una región pretende incrementar su nivel de ingresos más allá de los ingresos percibidos a través de bienes básicos o commodities, debe intensificar los esfuerzos en materia de innovación. Esta suele comenzar a pequeña escala, sin embargo, para que genere resultados de impacto es necesario que se extienda a toda la economía y beneficie por igual a empresas de diferentes sectores $y$ tamaños. A este proceso de le denomina difusión de la innovación.

Entre los trabajos empíricos que la relacionan con el crecimiento económico están los de Fagerberg (1994) y Verspagen (2001), que analizan el impacto del cambio tecnológico sobre el crecimiento económico y las diferencias internacionales en las tasas de crecimiento. Sus enfoques se enmarcan en la idea de entender la innovación como resultado de las actividades en $\mathrm{I}+\mathrm{D}$ intencionadas y desarrolladas por las empresas. Además, Verspagen (2001) separa el cambio tecnológico entre aquellas actividades relacionadas con la copia y absorción de tecnología foránea, de aquellas actividades que posicionan a un determinado país como líder tecnológico. Ambos tipos de actividades generan diferentes resultados en los patrones de convergencia entre las tasas de crecimiento económico de los países. Sener y Saridogan (2011) encuentran que los países con estrategias de competitividad global orientadas a la ciencia, la tecnología y la innovación tienen patrones de crecimiento económico a largo plazo, sostenible y competitivo (Baumert et al., 2016).

Estudios de las empresas más innovadoras y patentes de políticas han mostrado que hay una relación directa entre el compromiso del Estado a innovar, sus políticas fiscales en I + D y su habilidad de atraer y retener a organizaciones innovadoras. Estados Unidos y Japón, que poseen la mayor representación en la lista de Forbes de las 100 empresas más innovadoras, tienen una larga historia de estímulo gubernamental dirigido en este sentido (Forbes, 2013). Los incentivos varían según las necesidades y características propias de cada país. La idea es que sean diseñados para ajustarse a las necesidades de nuevas empresas y que consideren balancear el apoyo indirecto a la I + D por medio de incentivos tributarios con medidas de soporte directas para fomentar la innovación.

En el año 2020, el DANE publicó los resultados de la Encuesta de Desarrollo e Innovación Tecnológica aplicada a 
empresas del sector servicios y comercio (EDITS VII). Esta encuesta indaga sobre los productos, las actividades, los recursos y los instrumentos de apoyo asociados al desarrollo tecnológico y la innovación de las empresas del sector para los años 2018 y 2019 (DANE, 2020). Para la EDITS VII, el concepto de innovación es entendido como la introducción en el mercado de un producto (servicio o bien) nuevo o mejorado, o la implementación de un proceso nuevo o mejorado, que, según el Manual de Oslo, difieren significativamente de aquellos productos o procesos realizados previamente por la empresa (OCDE, 2018).

Entre algunos de los resultados que la EDITS VII presenta, se evidencia que en el ámbito nacional el subsector de telecomunicaciones registró en 2019 la mayor financiación de actividades científicas, tecnológicas y de innovación (ACTI) a través de recursos propios, con un monto de 1377530 millones de pesos; mientras que la mayor financiación con recursos públicos la registraron los centros de investigación y desarrollo con un monto de 261401 millones de pesos. A su vez, el estudio determinó que para el mismo periodo los principales obstáculos para realizar ACTI fueron el trámite excesivo y las condiciones de financiación poco atractivas para la obtención de recursos públicos. En Colombia, las empresas cuentan con diversas opciones para financiar sus proyectos, programas y actividades. Por una parte, se encuentran las fuentes de financiación que provee el Estado como parte de la política de apoyo empresarial $\mathrm{y}$, por otra, los recursos provenientes de la banca privada, otras empresas, fondos de capital privado, cooperación o donaciones y los recursos propios de la empresa. Aunque Colombia tuvo un buen desempeño innovador según el Global Innovation Index del año 2019, obtuvo el puesto 67 en el mundo sobre 141 economías; dentro del grupo de las economías catalogadas con ingreso medio-alto, quedó en la posición 18 sobre 38; para América Latina y el Caribe se posicionó en el sexto lugar (Cornell University et al., 2020). Según la OCDE (2014), el sistema de innovación en Colombia se sitúa por debajo de países de la región como Argentina, Brasil y Chile, los cuales lo superan en indicadores como el registro de patentes, las publicaciones científicas per cápita y el gasto en I+D. La Organización Mundial de la Propiedad Intelectual refuerza la idea de que la innovación puede contribuir al desarrollo de nuevas actividades económicas, potencializar la productividad y así mantener el aumento de los ingresos y la creación de empleo para la población urbana, fomentar la diversificación agropecuaria para mejorar el sustento de la población rural y mejorar la sostenibilidad medioambiental. Para esto es necesario que el sistema en Colombia refuerce el gasto bruto en $\mathrm{I}+\mathrm{D}$ con relación al PIB más allá del $0.2 \%$, que, comparado con el $1.2 \%$ de Brasil y el $2.4 \%$ de la OCDE, se queda realmente limitado con las proyecciones nacionales. 
Frente a esta problemática, como lo expone Parra (2011), el Gobierno colombiano en los últimos años se ha enfocado en materia de $\mathrm{I}+\mathrm{D}$, implementando una estrategia de incentivos económicos (deducciones fiscales $\mathrm{y}$ subvenciones), con el objetivo de posicionar al sector productivo en el centro del sistema de innovación nacional. Sin embargo, en Colombia los estudios abiertos al público que respalden la aceptabilidad y eficiencia de los incentivos son escasos. La Fundación Tecnos llevó a cabo una investigación titulada "Análisis del impacto de los proyectos del Sistema Nacional de Innovación", con el fin de dimensionar los resultados de proyectos de innovación, incluyendo los de cofinanciación, crédito directo e incentivos tributarios, en el periodo 1995-2000, que fueron realizados por empresas, en alianza empresa-universidad o empresa-centro de investigación y/o desarrollo tecnológico. De un total de 283 proyectos aprobados, el estudio aplicó 55 encuestas y se realizaron 37 entrevistas. Los principales hallazgos del estudio resaltaron que se obtuvieron 106 nuevos productos o servicios. El $80 \%$ de las empresas encuestadas tuvo mejoras en la producción y en la gestión. En el $45 \%$ de los proyectos se crearon o ampliaron nuevos negocios. La tasa de retorno de la inversión fue del $26 \%$ anual. Los productos derivados representan en promedio para las empresas el $27 \%$ de su participación en el mercado nacional. Se obtuvo un crecimiento del $29.7 \%$ de la productividad laboral. El $49 \%$ de las empresas desarrolló relacio- nes con proveedores, $37 \%$ con clientes y el $14 \%$ con competidores (Fundación Tecnos, citada por Colciencias, 2015).

Innovos Group, a través del programa "Locomotoras de Innovación", en el periodo evaluado 2011-2013 tenía como objetivo estimular procesos de innovación de producto, servicio o modelo de negocio, basados en conocimiento científico o de tecnología, mediante el acompañamiento de consultores internacionales a las empresas. Considerando una muestra de 91 empresas, 73 beneficiarias y 18 no beneficiarias, encontró que el $58 \%$ de los empresarios beneficiados manifestó mejoras en las ventas y el $44 \%$ afirmó percibir mejoras en su rentabilidad. El $100 \%$ de las empresas encuestadas considera haber acelerado la obtención de resultados de los proyectos gracias a la financiación obtenida de Colciencias. El análisis costo beneficio realizado para el $20 \%$ de las empresas beneficiarias, permitió identificar una tasa interna de retorno del $59 \%$ con un valor actual de los beneficios sociales netos de 7705 millones de pesos en el 2014 (Innovos Group, citado por Colciencias, 2015).

Finalmente, la investigación "Innovación y productividad en servicios: una evaluación de impacto de los programas de Colciencias en Colombia", de Umaña-Aponte et al. (2013) para 1999-2010, halló mejoras en la productividad laboral entre 23 y $26 \%$. Aunque actualmente no se discute el imprescindible papel del Gobierno en el fomento 
a la innovación, aún no hay una idea generalizada sobre cuáles políticas son más efectivas para lograr este objetivo.

\section{Instrumentos de fomento a la innovación}

El espíritu que orienta a los instrumentos de políticas públicas en materia de innovación, puestos en marcha por los gobiernos, es encontrar y llevar a cabo los cambios que propicien positivamente el cambio tecnológico y mantener o, en su caso, detonar de esta forma el crecimiento económico. Tal es la motivación por la que se pone en práctica este tipo de instrumentos por parte de las administraciones públicas de todo el mundo, desde luego, con resultados muy diversos. Primero surgen en países avanzados para luego implantarse prácticamente en todo el mundo.

\section{El Advanced Technology Programme} (ATP-Programa de Tecnología Avanzada), uno de los más importantes programas dirigidos al desarrollo tecnológico e innovador en los Estados Unidos, diseñado en 1988, se convierte en punto focal de la cooperación entre los sectores público y privado en torno al desarrollo de la innovación y la tecnología, así como para ayudar a resolver problemas importantes de los grandes segmentos de la industria (Avellar, 2007). Los beneficios se otorgan con base en los méritos técnicos y comerciales de cada proyecto. Proporciona, además, el capital semilla para empresas individuales, consorcios de universida- des, negocios o laboratorios de gobierno para desarrollo genérico, de tecnologías precompetitivas que tengan aplicaciones en la industria. El Programa Marco Europeo, Eureka, comenzó en 1985 y se mantiene hasta la actualidad en los países europeos. Consiste en una red europea de apoyo para el fomento de proyectos de investigación y desarrollo orientados al mercado, así como a la promoción de investigaciones colaborativas con el objeto de desarrollar innovaciones de productos y servicios. No constituye en sí un mecanismo de fondeo de recursos, por lo que no cuenta con financiamiento directo de la Unión Europea, pero sí se provee soporte financiero para el desarrollo del programa. Inicialmente, solo brindaba cobertura a las grandes empresas, pero desde el 2000 comenzó a ofrecerla a empresas más pequeñas (Gamboa-Quesada, 2014).

Los estudios que apoyan la efectividad de los incentivos tributarios para el fomento de la innovación eluden el hecho de que dichos incentivos no impactan de la misma manera a las empresas grandes y posicionadas que a las empresas pequeñas o emprendimientos, pues para las primeras es mucho más provechoso ese tipo de incentivos (Antolín et al., 2015). Esto se da, generalmente, porque las nuevas empresas en sus primeros años de operación no generan renta líquida, lo que ocasiona que los beneficios se apliquen solo en las empresas más grandes de determinados sectores, pues son las que poseen la capacidad financiera de soportar los diferentes 
incentivos (Departamento Nacional de planeación [DNP], 2015). La obtención de ayudas públicas ha sido reconocida como esencial en los primeros años de vida de la empresa, ya que es una forma de superar las limitaciones de recursos y capacidades a la hora de innovar (Verheul et al., 2009). Según Audretsch (2004), los emprendedores requieren de un contexto adecuado para llevar a cabo sus actividades económicas y el desarrollo de innovaciones. Por lo tanto, requieren ayudas públicas ajustadas a sus necesidades, que tengan en cuenta su potencial para promover el crecimiento económico y la innovación dentro de una región. Además, Bruton y Rubanik (2002) argumentan que las ayudas públicas pueden ser esenciales para la supervivencia y éxito de estas empresas.

Colombia ha desarrollado instrumentos para impulsar la innovación a través de líneas de crédito y cofinanciación, además de las convocatorias públicas para empresas interesadas en aplicar internamente estos procesos y desarrollo tecnológico, por ejemplo, el Ministerio de Ciencia, Tecnología e Innovación (Minciencias) destina recursos no reembolsables a través de convocatorias para el fomento de la investigación y la innovación dentro de las empresas. El Sistema General de Regalías (SGR) destina el $10 \%$ de los recursos para el Fondo de Ciencia, Tecnología e Innovación (FCTeI). INNpulsa Mipymes asigna recursos no reembolsables con la finalidad de fortalecer pequeñas y medianas empresas colombianas, y el Servicio Nacional de Aprendizaje (SENA) desde el año 2010 impulsa la innovación a través de la apertura de la convocatoria Fomento a la Innovación y el Desarrollo Tecnológico dentro de las empresas. Aunque el acceso de las empresas del departamento de Boyacá a este tipo de recursos es bajo, ha aumentado en los últimos años. Según la línea base de indicadores de ciencia y tecnología reportada por el Observatorio Colombiano de Ciencia y Tecnología (OCyT) entre 2008 y 2012 COLCIENCIAS financió 15 proyectos para Boyacá. Entre 2012 y 2015, el Fondo de Ciencia, Tecnología e Innovación aprobó cuatro proyectos por un monto de más de 57000 millones (OCyT, 2017). De igual forma, INNpulsa ha asignado más de 1800 millones a proyectos formulados en el departamento de Boyacá y el SENA a través de la convocatoria IDT Boyacá pasó de tener dos proyectos financiados entre los años 2010 y 2014 a cinco entre 2016 y 2017.

Los beneficios tributarios son un instrumento eficaz para fomentar la inversión en $\mathrm{I}+\mathrm{D}+\mathrm{i}$ y promover la competitividad en las empresas y el desarrollo de investigaciones de alto impacto para el país. Con el objetivo de calificar y definir los criterios y condiciones para el acceso a los beneficios tributarios, el Gobierno nacional creó el Consejo Nacional de Beneficios Tributarios, el cual está integrado por el ministro de Ciencia, Tecnología e Innovación, quién lo preside, el ministro de Hacienda y Crédito Público o el director de la Dirección 
de Impuestos y Aduanas Nacionales (DIAN) o su delegado, el ministro de Comercio, Industria y Turismo o su delegado, el ministro de Tecnologías de la Información y las Comunicaciones o su delegado, el director del Departamento Nacional de Planeación o su delegado, y dos expertos en ciencia, tecnología e innovación. Entre las principales ventajas se encuentran: $100 \%$ deducción sobre renta bruta de la inversión en I+D+i (Ley 1819 de 2016), máxima deducción por vigencia fiscal del $25 \%$ del impuesto de renta por pagar, independiente del tamaño de la empresa y de su sector de actividad, y cuando el descuento no pueda ser aplicado en su totalidad en la vigencia fiscal correspondiente, el excedente podrá utilizarse en los siguientes cuatro años.

Los principales resultados del Índice Departamental de Innovación para Colombia (IDIC) situaron a Boyacá en 2019 en el puesto 11 entre 31 departamentos. Ocupa el puesto tres dentro del grupo de desempeño medio. A esto contribuyen sus buenos resultados en el pilar de capital humano e investigación, en el que ocupa el puesto 4 entre todos los departamentos, gracias a la cobertura y calidad sobresaliente en los distintos niveles educativos. Los indicadores en los que muestra mayor rezago son gasto en TIC para innovar (puesto 23), inversión en TIC en empresas que introducen nuevos métodos organizacionales (puesto 19), producción en economía naranja (puesto 17) y registro de software (puesto 16). Lo anterior nos muestra que, en términos generales, en Boyacá la producción de tecnología y bienes y servicios creativos es aún muy incipiente. Sin embargo, el mayor retroceso (-15 posiciones) ocurrió en un indicador del pilar de instituciones. De igual forma, otros estudios han logrado identificar que las empresas boyacenses presentan bajos niveles de innovación que limitan su acceso a nuevos mercados, provocan estancamiento en sus procesos productivos y originan a medio y largo plazo una reducción de los beneficios económicos y sociales (DNP, 2019).

\section{METODOLOGÍA}

En la dinámica empresarial cada vez adquiere más relevancia la metodología cualitativa, debido a la permanente necesidad del tipo de información obtenida a través de la misma (Martínez, 2006), además de la potencialidad de entender el contexto, su flexibilidad y su capacidad de captar elementos subjetivos invisibles para los indicadores cuantitativos (Taylor \& Bogdan, 1989).

Esta es una investigación con enfoque cualitativo, de tipo descriptivo, a través del estudio de casos. Aunque existen varias definiciones para los estudios de caso, para Eisenhardt (1989, p. 4) es "una estrategia de investigación dirigida a comprender las dinámicas presentes en contextos singulares". Ruiz (2002), por su parte, considera el estudio de casos como el análisis de un incidente, situación o hecho en concreto mediante 
la recopilación de información selectiva que permite al investigador entender los elementos de una determinada situación.

Se plantearon tres etapas. En primer lugar se revisó la literatura existente sobre los instrumentos públicos de fomento a la innovación empresarial en el nivel nacional y departamental (Tabla 1); para el diseño del estudio de caso se siguió la metodología propuesta por Yin (2009) a través del diseño del estudio, conducción del caso, preparación, recolección de datos, análisis y elaboración del reporte de estudio.

Tabla 1. Documentos públicos revisados

\begin{tabular}{|c|c|c|c|}
\hline Año & Nombre & Descripción & Origen \\
\hline 2009 & Ley 1286 de 2009 & Ley de Ciencia Tecnología e Innovación (CTel) & \multirow{10}{*}{$\begin{array}{c}\text { Congreso de } \\
\text { la República } \\
\text { de Colombia y } \\
\text { Departamento } \\
\text { Nacional de } \\
\text { Planeación }\end{array}$} \\
\hline 2009 & Conpes 3582 de 2009 & Política Nacional de CTel & \\
\hline 2010 & $\begin{array}{l}\text { Plan Nacional de } \\
\text { Desarrollo 2010-2014 }\end{array}$ & $\begin{array}{l}\text { Innovación como una de las } \\
\text { cinco "locomotoras" }\end{array}$ & \\
\hline 2010 & Conpes 3668 de 2010 & $\begin{array}{l}\text { Seguimiento a la Política Nacional de } \\
\text { Competitividad y Productividad }\end{array}$ & \\
\hline 2011 & Acto Legislativo 06 de 2011 & Fondo de CTI del SGR & \\
\hline 2012 & Ley 1530 de 2012 & $\begin{array}{l}\text { Regulación y funcionamiento del } \\
\text { Sistema General de Regalías }\end{array}$ & \\
\hline 2012 & Decreto 1500 de 2012 & $\begin{array}{c}\text { Sistema Nacional de Competitividad } \\
\text { e Innovación (SNCl) }\end{array}$ & \\
\hline 2015 & Ley 1753 de 2015 & $\begin{array}{l}\text { Plan Nacional de Desarrollo 2014- } \\
2018 \text { "Todos por un nuevo país" }\end{array}$ & \\
\hline 2015 & Conpes 3834 de 2015 & $\begin{array}{c}\text { Lineamientos de política para estimular } \\
\text { la inversión privada en CTel a través } \\
\text { de deducciones tributarias }\end{array}$ & \\
\hline 2015 & $\begin{array}{l}\text { Plan Nacional de } \\
\text { Desarrollo 2014-2018 }\end{array}$ & $\begin{array}{l}\text { Articulación del SNCTel al Sistema } \\
\text { Nacional de Competitividad }\end{array}$ & \\
\hline $\begin{array}{l}2012- \\
2022\end{array}$ & PEDCTI & $\begin{array}{l}\text { Plan Estratégico Departamental de Ciencia, } \\
\text { Tecnología e Innovación Boyacá }\end{array}$ & \multirow{4}{*}{$\begin{array}{c}\text { Gobernación } \\
\text { de Boyacá }\end{array}$} \\
\hline 2017 & $\begin{array}{l}\text { Banco de Programas y } \\
\text { Proyectos de Inversión } \\
\text { Nacional -BPIN }\end{array}$ & $\begin{array}{l}\text { Implementación de una convocatoria de } \\
\text { proyectos de i+d para el desarrollo tecnológico } \\
\text { de base biológica que contribuyan a los } \\
\text { retos del departamento de Boyacá }\end{array}$ & \\
\hline 2017 & BPIN & $\begin{array}{l}\text { Implementación de un sistema de gestión } \\
\text { de innovación para la industria de la región } \\
\text { central Boyacá: Innovación más país Boyacá }\end{array}$ & \\
\hline 2018 & BPIN & $\begin{array}{c}\text { Desarrollo estratégico agroecológico } \\
\text { para el fortalecimiento del } \\
\text { sector productivo en el } \\
\text { departamento de Boyacá }\end{array}$ & \\
\hline
\end{tabular}


Continuación Tabla 1

\begin{tabular}{|c|c|c|c|}
\hline $\begin{array}{l}2014- \\
2018\end{array}$ & $\begin{array}{c}\text { Plan estratégico } \\
\text { institucional } \\
\text { Colciencias 2014-2018 }\end{array}$ & Estrategia institucional & Colciencias \\
\hline 2015 & $\begin{array}{c}\text { Programas nacionales } \\
\text { del SNCTel }\end{array}$ & Programas generales para la CTel & \\
\hline 2013 & INNpulsa Mipyme & $\begin{array}{l}\text { Fomipyme se vincula a INNPULSA } \\
\text { bajo el nombre de iNNpulsa Mipyme }\end{array}$ & iNNpulsa \\
\hline $\begin{array}{l}2010- \\
2014\end{array}$ & SENA IDT & $\begin{array}{l}\text { Fomento de la Innovación y el } \\
\text { Desarrollo Tecnológico }\end{array}$ & \\
\hline $\begin{array}{l}2015- \\
2017\end{array}$ & SENA IDT & $\begin{array}{l}\text { Fomento de la Innovación y el } \\
\text { Desarrollo Tecnológico en las } \\
\text { Empresas, con enfoque regional }\end{array}$ & SENA \\
\hline
\end{tabular}

Fuente: elaboración propia.

En segundo lugar, y con el objetivo de obtener información de análisis, se aplicaron entrevistas semiestructuradas a dos empresas beneficiarias de la convocatoria pública del Sena IDT "Fomento de la Innovación y el Desarrollo Tecnológico en la Empresas 2015-2017, con enfoque regional", en su proceso II. La cual buscaba aportar al fortalecimiento del sector productivo de la región, a través de la financiación de proyectos de innovación y desarrollo tecnológico, y de la generación de capacidades en las empresas, para la innovación, formulación y presentación de proyectos que impactan la productividad y competitividad regional. Se llevó a cabo a través de tres líneas programáticas a) desarrollo e implementación de tecnologías o conocimientos que se originen en las empresas, o a partir de investigación aplicada, en el que se creen nuevos productos, servicios o la generación de nuevos procesos, b) Transferencia o apropiación de tecnologías o cono- cimientos que mejoren la capacidad competitiva, la gestión tecnológica y la innovación de productos y procesos en las empresas y c) apoyo de iniciativas de innovación y desarrollo tecnológico que se originen en los diferentes sectores productivos y que se enmarquen en el diseño, apropiación y desarrollo de nuevas prácticas de acuerdo con los estándares internacionales.

El presupuesto total de la convocatoria para el proceso II fue de 18444492 860 pesos y específicamente para la región Centro Oriente (departamentos de Boyacá, Cundinamarca, Norte de Santander, Santander y Bogotá, D. C.) fue de 4780000000 pesos. El aporte máximo de cofinanciación para la asignación de recursos no reembolsables fue del $50 \%$ del valor total del proyecto para empresas grandes y un $80 \%$ para proyectos de empresas micro, pequeñas y medianas. Respecto al monto, 
el valor máximo para cofinanciar por parte del SENA fue de 200000000 de pesos por proyecto.

Es importante destacar que, aunque el objetivo del modelo de medición es poder ser aplicable a un grupo significativo de empresas, para el desarrollo de la investigación fue aplicado a dos mipymes debido a la profundidad del mismo y a la escasez de empresas beneficiarias de recursos públicos para el fomento de la innovación en el departamento de Boyacá, que para el periodo de análisis comprendidito entre el 2015 y 2017 fue de cuatro empresas (Servicio Nacional de Aprendizaje [SENA], 2017).

Tabla 2. Empresas participantes en el estudio de caso

\begin{tabular}{cccc}
\hline Empresa & Representante legal & Actividad económica & Código CIIU \\
\hline MAGICC FOOD S.A.S. & Sergio Leonardo Venegas Salcedo & $\begin{array}{c}\text { Elaboración de } \\
\text { productos de panadería }\end{array}$ & 1081 \\
PROYECSOFT S.A.S. & José Ángel Puentes Osorio & Desarrollo de software & 6201 \\
\hline
\end{tabular}

Fuente: elaboración propia.

El instrumento se aplicó en el segundo semestre del 2019 directamente a los líderes del proyecto beneficiario, quienes fueron los encargados de validar su ejecución durante el transcurso del 2018. Ellos conocían en profundidad los impactos generados por la innovación realizada y eran, a su vez, los representantes legales de las empresas. El instrumento de medición integró simultáneamente el desarrollo de una entrevista y la aplicación de una encuesta semiestructurada, dividida en nueve secciones en donde se buscó extraer información general de la empresa, las actividades de $\mathrm{I}+\mathrm{D}$ interna relevantes, las actividades para la innovación, las innovaciones de productos y de procesos, los factores que dificultan las actividades de innovación, los derechos de propiedad intelectual e industrial, las innovaciones no tecnológicas, las innovaciones de comercialización y el financiamiento público.

\section{RESULTADOS}

Se analizaron las respuestas dadas por las empresas Magicc Food S.A.S. y Proyecsoft S.A.S. en cada una de las nueve secciones del instrumento. Inicialmente, se identifica que son empresas "nuevas", pues fueron constituidas legamente en el año 2012 y 2013 respectivamente. Percibieron un significativo aumento en las ventas y, para el caso de Magicc Food, una ampliación en los canales de comunicación con los clientes logró una mayor participación en el mercado. Adicionalmente, manifiestan que durante la ejecución 
del proyecto lograron generar nuevos empleos, los cuales se mantienen debido a la implementación de la innovación y se resalta el particular interés que se generó en los trabajadores hacia una cultura de la innovación y la formulación de proyectos.

\section{Actividades de I+D interna}

Para esta sección y a causa de la disparidad en la actividad económica de las empresas objeto de estudio, se evidenció que Magicc Food centra sus esfuerzos en el desarrollo y fortalecimiento de otras áreas de la empresa y, por el contrario, Proyecsoft participa activamente en la generación de trabajos creativos que fomentan la I+D interna. Las dos empresas coinciden en manifestar la importancia de desarrollar actividades de I+D interna y en el impulso que la inyección de recursos públicos representa para tal fin.

\section{Actividades para la innovación}

Para la pregunta C.3 iSi su empresa no hubiera recibido apoyo financiero público (préstamos, subvenciones...) para las actividades de innovación tecnológica durante el año 2018, las habría realizado? Aunque las respuestas manifiestan un enfoque diferente, convergen en la necesidad de un apalancamiento financiero por parte del Estado debido a su tamaño y a su capacidad operativa. Además, reconocen un mercado cada vez más competitivo y a los procesos de innovación como un factor diferenciador de éxito.

\section{Fuentes de información para activi- dades de innovación}

Se logró determinar que dentro de las fuentes de información más significativas para desarrollar actividades de innovación se encuentra, y con un grado de "extrema importancia", la información derivada dentro de la empresa, seguida de las fuentes del mercado a través de sus competidores $u$ otras empresas de su misma actividad y por medio de consultores, laboratorios comerciales o institutos privados de I+D. En contraste a lo esperado, las universidades, las publicaciones en revistas científicas/técnicas/comerciales y los eventos académicos quedan rezagados por los empresarios, lo que evidencia la desarticulación de la triada universidad-Estado-empresa.

\section{Objetivos de la innovación}

Para esta pregunta se clasificaron los objetivos teniendo en cuenta su orientación a productos, procesos, empleo, financieros y otros. Fueron calificados de extremada importancia los relacionados con la penetración en nuevos mercados, mayor calidad de los bienes y servicios, menores costos laborales por unidad producida, mantenimiento del empleo, incremento de ingresos y el incremento patrimonial. 
Factores que dificultan las actividades de innovación

Según las respuestas dadas por los empresarios, se identificó que los factores más influyentes para limitar o dificultar las actividades de innovación son la falta de fondos económicos en la empresa, la falta de financiación de fuentes exteriores, los costos elevados en los procesos de innovación y la falta de personal cualificado. Particularmente, una de las empresas manifestó la dificultad para encontrar socios de cooperación para la innovación arraigados en la cultura regional en el departamento de Boyacá, así como la incertidumbre respecto a la demanda de bienes y servicios innovadores.

\section{Derechos de propiedad intelectual e industrial}

Aunque los empresarios manifiestan reconocer la importancia de los procesos de PI, el panorama actual nacional y regional demuestra el rezago significativo que existe en la solicitud y obtención de patentes, registros de marcas y protección de derechos de autor. Sin embargo, resaltan que los procesos de innovación generan un efecto de "bola de nieve" al ver la necesidad de proteger los resultados obtenidos con la ejecución del proyecto financiado por la convocatoria SENA IDT. Actualmente adelantan los trámites necesarios para obtener dichos derechos y los beneficios que conllevan.
Finalmente, para la sección Financiamiento público se concluye, según las respuestas dadas por las empresas objeto de estudio, que el apoyo del Estado es clave para impulsar $\mathrm{y}$ fomentar los procesos de innovación en el departamento. Sin embargo, la falta de acceso a la información de convocatorias disponibles se convierte en un limitante trascendental en el proceso; además, la escasa cultura hacia la innovación que presenta el departamento hace que los programas que desarrolla el gobierno regional parezcan deficientes en el ámbito nacional.

Los resultados obtenidos de esta investigación demuestran que, pese a ser evidente la importancia de la inversión pública de recursos en el fomento de la innovación empresarial en el departamento de Boyacá, su eficacia se ve limitada porque, aunque aumenta sustancialmente el número de innovaciones en productos, procesos y métodos empresariales dentro de la empresa, no se está contribuyendo a la generación de nuevos productos, sino a la adaptación de tecnologías externas. Además, se puede determinar que el nivel de innovación en las empresas es directamente proporcional a su tamaño, su estructura productiva, su capacidad financiera y cultura hacia la innovación por medio de inversión en actividades de I+D.

Algunas de las recomendaciones derivadas del análisis de los resultados obtenidos demuestran la necesidad de articular la información generada por 
los centros de investigación y las universidades con el tejido empresarial del departamento, con el fin de dinamizar y aunar esfuerzos hacia una cultura de innovación relevante. De igual forma, es necesario el apalancamiento de empresas en desarrollo mediante la eliminación de barreras y la promoción de la expansión del emprendimiento para generar nuevas ideas; el fortalecimiento del capital humano de calidad, con el fin de evitar la fuga de perfiles hacia otros departamentos; y finalmente, socializar la importancia de los procesos de PI como una estrategia de retorno a la inversión.

\section{CONCLUSIONES}

El objetivo principal de este trabajo fue analizar la influencia de los recursos públicos en el fomento de la innovación empresarial del departamento de Boyacá y aportar información significativa a los actores que intervienen en todo el ecosistema innovador de la región. Los resultados sugieren que las empresas del departamento que acceden a recursos públicos lo hacen en primera instancia para incrementar sus niveles de innovación interna, sin que su principal propósito sea generar innovaciones en el mercado nacional o internacional. Sin embargo, el análisis de la investigación también permitió identificar que más allá de buscar un apoyo financiero para generar innovaciones en productos o servicios, es necesario articular cada una de las actividades que intervienen en los procesos innovativos, como la inversión en investigación y desarrollo (I+D), un sistema educativo de calidad, voluntad política de crecimiento y desarrollo a través de actividades de CTeI, además de fomentar estrategias para la articulación de universidad-Estado-empresa.

El análisis de los casos de éxito citados permite mencionar que las empresas que son sometidas a procesos de innovación de producto, servicio o modelo de negocio, actualmente dedican hasta un $112.8 \%$ más del presupuesto tradicionalmente asignado para actividades de innovación en la empresa, tienen $86.8 \%$ más proyectos o programas relacionados con la innovación, registran $154.7 \%$ más patentes, vinculan $26 \%$ más empleados profesionales, vinculan $43.8 \%$ más empleados con posgrado, entre otros beneficios.

Según datos de la Contraloría General de la República (2014), los incentivos económicos para la innovación son empleados en Colombia desde los años noventa, sin embargo, no existen evaluaciones que permitan medir su impacto e influencia. En relación con lo anterior, el objetivo de esta investigación es aportar información sobre la influencia de la inversión de recursos públicos en el fomento de la innovación empresarial y el crecimiento económico en el departamento de Boyacá. 
Finalmente, se considera que este departamento presenta actualmente las condiciones necesarias (altos niveles académicos, enfoque hacia la innovación, disponibilidad de recursos, etc.) para avanzar en un proceso de crecimiento económico que genere un aporte significativo al desarrollo regional y nacional. $\mathrm{Su}$ enfoque ahora debe ser engranar las piezas iniciales de una explosión creativa de nuevos productos, nuevos procesos, nuevos horizontes comerciales, sociales y de conocimiento.

\section{CONTRIBUCIÓN DE LOS} AUTORES

El trabajo es el resultado del trabajo conjunto de los autores.

\section{FINANCIAMIENTO}

Los autores agradecen el apoyo financiero de la Vicerrectoría de Investigación y Extensión de la UPTC con el Proyecto SGI 2553 - Influencia de la inversión de recursos públicos en el fomento de la innovación empresarial en el departamento de Boyacá. Derivado de la convocatoria Jóvenes Investigadores.

\section{AGRADECIMIENTOS}

Los autores agradecen a las dos empresas participantes en el estudio, Magicc Food S.A.S. y Proyecsoft S.A.S, así como a los pares evaluadores por sus observaciones.

\section{DECLARACIÓN CONFLICTO DE INTERESES}

Los autores declaran que no existe conflicto de intereses. 


\section{REFERENCIAS}

[1] Antolín, R., Céspedes, J. \& Martínez, J. (2015) Fomentando la innovación de producto en las empresas nuevas: ¿Qué instrumentos públicos son más efectivos? Investigaciones Europeas de Dirección y Economía de la Empresa, 22(1), 38-46. http://doi.org/10.1016/j.iedee.2015.05.002.

[2] Arrow, K. J. (1962) Economic Welfare and the Allocation of Resources for Invention. In NBR, The Rate and Direction of Inventive Activity: Economic and Social Factors (pp. 609-626). NBR http://www.nber.org/books/univ62-1. https:// doi.org/10.1515/9781400879762-024

[3] Audretsch, D. B. (2004). Sustaining Innovation and Growth: Public Policy Support For Entrepreneurship. Industry and Innovation, 11(3), 167-191. https://doi. org/10.1080/1366271042000265366

[4] Avellar, A. P. M. (2007). Metodologías de evaluación de políticas tecnológicas: reseña de prácticas internacionales. Cepal, 1-43. http://www.cepal.org/iyd/noticias/paginas/0/31430/metdeevalua.pdf.

[5] Baumert, T., Buesa, M., Gutiérrez, C. \& Heijs, J. (2016). Innovación y crecimiento económico. Instituto de Análisis Industrial y Financiero.

[6] Bruton, G. D. \& Rubanik, Y. (2002). Resources of the Firm, Russian High-Technology Startups and Firm Growth. Journal of Business Venturing, 17(6), 553-566. https://doi.org/10.1016/S0883-9026(01)00079-9

[7] CEPAL.(2006). Acerca de innovación, ciencia ytecnología. https://www.cepal.org/ es/temas/innovacion-ciencia-y-tecnologia/acerca-innovacion-ciencia-tecnologia.

[8] Colciencias. (2015). Experiencias en evaluación de ciencia, tecnología e innovación. Colciencias.

[9] Contraloría General de la República. (2014). Evaluación política pública, beneficios tributarios ciencia, tecnología e innovación. Contraloría General de la República.

[10] Cornell University, INSEAD \& WIPO. (2020). The Global Innovation Index 2020: Who Will Finance Innovation? Itaca.

[11] DANE. (2020). Encuesta de Desarrollo e Innovación Tecnológica, 2018-2019. DANE. 
[12] Departamento Nacional de Planeación -DNP-. (2015). Lineamientos de política para estimular la inversión privada en ciencia, tecnología e innovación a través de deducciones tributarias. https://colaboracion.dnp.gov.co/CDT/Conpes/Econ\%$\mathrm{C} 3 \% \mathrm{~B} 3 \mathrm{micos} / 3834$.pdf

[13] Departamento Nacional de Planeación -DNP-. (2019). Índice Departamental de Innovación para Colombia (IDIC). https://colaboracion.dnp.gov.co/CDT/Prensa/ Indice-Departamental-Innovacion-Colombia-2019.pdf.

[14] Eisenhardt, K. M. (1989). Building Theories from Case Study Research. Academy of Management Review, 14(4), 532-550.

[15] Fagerberg, J. (1994). Technology and International Differences in Growth Rates. Journal of Economic Literature, 32.

[16] Forbes. (2013). Is Innovation Inspired by Tax Policy? Revista Forbes. http://www. forbes.com/sites/joeharpaz/2013/10/11/isinnovation-inspired-by-tax-policy/.

[17] Gamboa-Quesada, F. (2014). La importancia del marco teórico de la innovación tecnológica en el diseño de políticas públicas y el comportamiento de instrumentos relevantes de esta materia en México. Universidad Nacional Autónoma de México.

[18] Martínez, P. C. (2006). El método de estudio de caso. Estrategia metodológica de la investigación científica. Pensamiento y Gestión, (20), 165-193.

[19] OCyT (2017). Línea Base de Indicadores de Ciencia y Tecnología. Boyacá. Bogotá: Observatorio Colombiano de Ciencia y Tecnología. https://oi. org/10.25054/01247905.1377

[20] Organización de Cooperación y Desarrollo Económicos -OCDE-. (2014). Estudios de la OCDE de las políticas de innovación: Colombia Resumen ejecutivo. https://www.oecd.org/sti/inno/colombia-innovation-review-assessment-and-recommendations-spanish.pdf.

[21] Organización de Cooperación y Desarrollo Económicos -OCDE-. (2018). Manual de OSLO (4.a ed.). Guidelines for Collecting, Reporting and Using Data on Innovation. OECD Publishing.

[22] Parra, M. (2011) Exenciones fiscales para la I+D+i Experiencias en América Latina y retos pendientes. BID. https://publications.iadb.org/handle/11319/5690.

[23] Romer, P. M. (1990). Endogenous Technological Change. Journal of Political Economy, 98(5), S71-S102. http://doi.org/10.1086/261725. 
[24] Ruiz, M. (2002). Técnicas Cualitativas de Investigación Educativa. Notas del curso. Comunicación electrónica personal. Universidad ORT.

[25] Schumpeter, J. A. (1939). Business Cycles: A Theoretical, Historical, and Statistical Analysis of the Capitalist Process. Volumen 1. Martino Pub.

[26] Sener, S. \& Saridogan, E. (2011). The effects of science-techonology-innovation on competitiveness and economic growth. Procedia Social and Behavioral Sciences, 24, 815-828. https://doi.org/10.1016/j.sbspro.2011.09.127

[27] Servicio Nacional de Aprendizaje -SENA-. (2017). Pliego definitivo Convocatoria de Fomento de la Innovación y el Desarrollo Tecnológico en las Empresas, con enfoque Regional 2015-2017, Proceso II. http://www.tecnnova.org/wp-content/ uploads/2016/06/PrePliego_TerminosEstrategia2015-17_Proceso_I.pdf

[28] Taylor, S. \& Bogdan, R.C. (1989). Introducción a los métodos cualitativos de investigación. Paidós.

[29] Umaña-Aponte, M., Estupiñán, F. \& Duque, C. (2013). Innovation and Productivity in Services: An impact evaluation of Colciencias funding programs in Colombia. Métrica Investigaciones y Proyectos.

[30] Verheul, I., Carree, M. \& Santarelli, E. (2009). Regional Opportunities and Policy Initiatives for New Venture Creation. International Small Business Journal, 27(5), 608-625. https://doi.org/10.1177/0266242609338757

[31] Verspagen, B. (2001). Economic growth and technological change: an evolutionary interpretation. STI Working Papers 2001/1. OECD.

[32] Yin, R. K. (2009). Case Study Research: Design and Methods. Sage Publications. 